\title{
Protocolo para el diseño de exámenes extraordinarios
}

Alejandra Torres Landa López, Gabriela Ramos Solórzano y Adriana Morales Mireles

\section{Resumen}

$\mathrm{E}$ ste artículo es resultado de un miniproyecto ${ }^{1}$ de investigación denominado "Protocolo para el diseño de exámenes extraordinarios". El propósito es contar con criterios para el diseño e implementación de los exámenes extraordinarios en las materias del Departamento de Teoría y Métodos del Centro de Ciencias del Diseño y de la Construcción de la Universidad Autónoma de Aguascalientes [UAA]. Se partió del diagnóstico aleatorio del estado actual de los exámenes extraordinarios como instrumentos de medición para la evaluación del aprendizaje de los estudiantes, cuando éstos no han logrado alcanzar una calificación aprobatoria al final de un curso. Se tuvo como referentes institucionales el Modelo Educativo Institucional [MEI] y el Reglamento General de Docencia de la UAA [RGD]. La investigación se argumenta en la necesidad de contar con un diseño de calidad de exámenes extraordinarios en el área académica que, de manera objetiva, puntual y completa, tome en cuenta los contenidos tanto cuantitativos como cualitativos de formación de una materia en particular.

Palabras clave: protocolo de exámenes, criterios de evaluación, exámenes extraordinarios, evaluación de los aprendizajes, procedimientos para exámenes.

\section{Introducción}

El MEI señala que la evaluación es una dimensión de los procesos de enseñanza y aprendizaje, el cual da cuenta del logro de los objetivos de formación; es instrumentada en distintos momentos del proceso educativo, pudiendo ser una evaluación diagnóstica, procesual y sumativa, que se utiliza para conocer el desempeño de los estudiantes, contar con elementos

1 Los mini-proyectos tienen el propósito de fortalecer las habilidades para la investigación y contribuir a la vocación científica, a través del desarrollo semestral de un tema de interés en el ámbito educativo y profesional. En el Centro de Ciencias del Diseño y de la Construcción (ccDyc) se lleva a cabo en dos modalidades: para estudiantes, con el apoyo de un profesor-asesor, y para profesores. para reorientar el proceso de enseñanza, hacer mejoras que conduzcan al logro de los propósitos educativos, identificar ajustes potenciales a los objetivos del currículo y detectar deficiencias en las prácticas mismas de evaluación (UAA, 2007). Las metodologías que se empleen para la evaluación de los aprendizajes deben ser congruentes con los enfoques de la enseñanza, así como con las estrategias e instrumentos que sirvan a los profesores para evaluar el progreso de aprendizaje de sus estudiantes; quienes participan activamente a través de la coevaluación y autoevaluación (UAA, 2007).

《Los exámenes extraordinarios son una oportunidad para los estudiantes; velar por la calidad en el diseño,

proceso e implementación de los mismos es una responsabilidad del profesorado.»

Las características de los exámenes extraordinarios deben ajustarse a lo establecido en el programa de materia, tal como lo señala el Art. 29 del RGD (UAA, 2018), donde el grupo de profesores expertos adscritos a un área es responsable de establecer y definir criterios para el diseño e implementación de los exámenes extraordinarios, tomando en cuenta la naturaleza del conocimiento, el programa de la materia y el desarrollo metodológico en el aula, logrando así un proceso completo y claro, al mismo tiempo que un diseño adecuado del examen extraordinario, en cuanto a complejidad, ponderación, extensión, duración y objetividad; de manera consensuada y aprobada por los integrantes de la academia.

Un diseño adecuado de los exámenes extraordinarios contribuye a que los estudiantes decidan con mayor responsabilidad solicitarlo y presentarlo, se comprometan a prepararse de la mejor manera que les sea posible y logren aprobar con calificación satisfactoria su examen. Los exámenes extraordinarios son una oportunidad para los estudiantes; velar por la calidad en el diseño, proceso e implementación de los mismos es una responsabilidad del profesorado, por tanto, se deben señalar, consensar y aprobar los criterios que deberán observarse para mantener y mejorar el desempeño de los educandos. 
La concepción que los profesores tengan sobre la educación y la evaluación es un aspecto determinante que puede ayudar u obstaculizar el proceso de los exámenes extraordinarios. Lo señalado en el MEI es la base que consolida y orienta la propuesta que se presenta; aunque también se toman en consideración las siguientes premisas, descritas por Bertoni, Poggi y Teobaldo (2000), respecto a la evaluación de los aprendizajes:

1. Es imperante evaluar procesos y no solamente resultados; cuando un estudiante presenta un examen extraordinario, algo ha aprendido previamente, esto debe ser tomado en cuenta por él y por el profesor como algo imperioso.

2. Es necesario evaluar no sólo conocimientos; las actitudes son sumamente importantes desde una visión integral de formación, éstas pueden ser un factor determinante en la evaluación del estudiante.

3. Es importante incluir en la evaluación los resultados previstos y los no previstos; así como los efectos observables, los no observables y los implícitos.

La investigación llevó a diagnosticar, en un primer momento, el estado actual de los programas de materia del área, bajo la perspectiva de Wiggins y McTighe (2005) sobre el diseño a la inversa, que propone: identificar los resultados que se esperan, determinar la evidencia aceptable o evaluable y, en consecuencia, planificar las experiencias de aprendizaje. Lo anterior llevó a plantear el Esquema 2.

Acorde con lo anterior, se diseñaron dos instrumentos: una cédula de registro y una lista de cotejo. ${ }^{2} \mathrm{El}$ primero para identificar los contenidos más importantes de los programas de materia, su ponderación y la manera en que se evaluaron los aprendizajes; lo que conformó, con el segundo, la base para el análisis de los exámenes extraordinarios.

\section{«La razón de ser de la evaluación es servir a la acción educativa, entendida desde una visión integral.〉}

2 La cédula de registro y la lista de cotejo utilizadas podrán solicitarse directamente a la dirección de correo de las coordinadoras de academia: Alejandra Torres Landa (alejandra. torreslanda@edu.uaa.mx) y Adriana Morales Mireles (adriana. morales@edu.uaa.mx).
Esquema 1. Gestión de los contenidos del programa de materia para el diseño a la inversa de las experiencias de aprendizaje

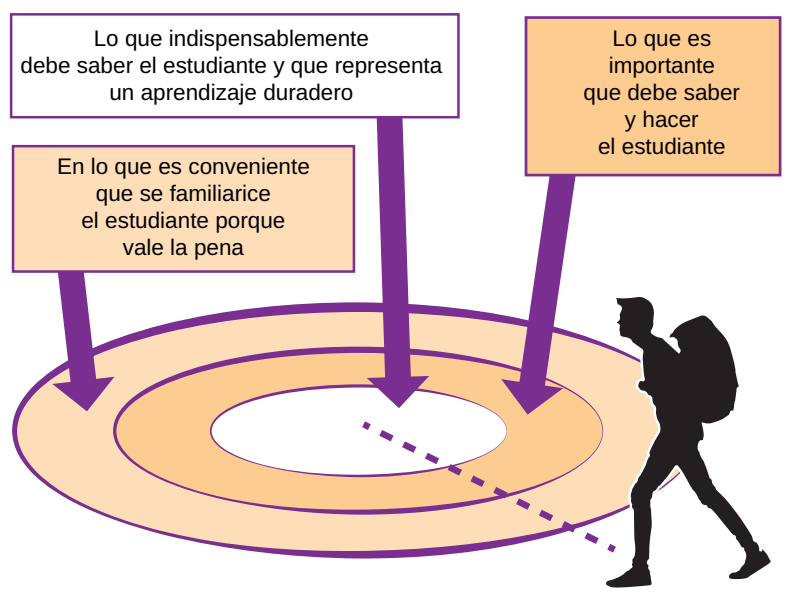

Fuente: elaboración de las autoras, a partir de Wiggins, G. y McTighe, J. (2005). (Cap. 1).

\section{Resultados}

Un problema generalizado que se identificó en los programas de materia fue que los objetivos generales y particulares no siempre son claros. Los criterios de evaluación de los aprendizajes señalados en los programas de materia son redactados en términos muy generales, no hay especificaciones que permitan conocer otros aspectos importantes, como las características del examen, el tipo de evaluación, su ponderación, momento de la evaluación y la evidencia aceptable. Por su parte, los exámenes extraordinarios, en su mayoría, tienen poca especificación y relación ponderada con los contenidos del programa de materia, pues muestran poca variedad de reactivos y niveles de complejidad.

En este sentido, la propuesta de "Protocolo para el diseño de exámenes extraordinarios" considera que todo diseño de examen, trabajo o proyecto integral que funja como tal, debe abarcar los siguientes criterios:

1. Partir del análisis previo del programa de la materia (cédula de registro) y del análisis previo del examen extraordinario existente (lista de cotejo) en su caso.

2. Contar con elementos de identificación suficientes, como: logotipo oficial de la institución, departamento, academia, nombre del profesor/es que diseñó el instrumento de evaluación, año en que se diseñó y fecha de aprobación por la academia. También el nombre de la materia, carrera, semestre, objetivo general, maestro 
que aplica, nombre e Id. del estudiante, fecha en que se realiza la evaluación y tiempo límite del examen.

3. Contener instrucciones claras, suficientes y precisas en cada uno de sus apartados (Flore y García, 2018).

4. Contemplar la totalidad de los contenidos del programa de la materia que se evalúa, tomando en cuenta el valor porcentual de cada una de las unidades; en ningún caso aplican puntos extras o preguntas de salvamento (Coordinación de Calidad Académica, 2014).

5. El trabajo o proyecto integral que se aplique como examen extraordinario debe contar con una rúbrica clara y completa de evaluación; se sugiere contemplar la participación de un profesor del área como invitado en el proceso (Coordinación de Calidad Académica, 2014).

6. El examen extraordinario, dependiendo de sus características, debe ser ejecutado durante los días necesarios, en el espacio físico adecuado para ello y siempre monitoreado por el/los profesor/es designado/s.

7. El examen extraordinario se debe elaborar de manera que el estudiante demuestre sus conocimientos y habilidades, tanto en el aspecto teórico como en el práctico.

8. El examen debe estar diseñado, por lo menos, con cuatro tipos diferentes de reactivos, en correspondencia con la naturaleza de los contenidos a evaluar y tomando en cuenta algunos de los elementos de los exámenes parciales aplicados.

9. El profesor que impartió la materia debe dar, de antemano, la evaluación de sus actitudes ${ }^{3}$ a los estudiantes que soliciten el extraordinario; misma que se sumará en el porcentaje acordado por la academia a la calificación total del examen.

10. La academia debe trabajar en sugerencias y lineamientos para el diseño de reactivos de evaluación del área, con el fin de considerarse como estándares de calidad en el diseño de los instrumentos de evaluación.

11. Todo examen que se diseñe y apruebe en la academia debe pasar a una base de datos por área, tomando en cuenta los derechos de autoría que la academia acuerde.

\section{Reflexiones finales}

Como señala Suárez e Hinojoza (2010), la razón de ser de la evaluación es servir a la acción educativa, entendida desde

3 La evaluación de las actitudes deberá evidenciarse con base en el registro de las mismas durante el semestre, las actitudes a evaluar en cada materia serán previamente aprobadas en academia tomando en cuenta la naturaleza de la misma y su contribución a perfil de egreso; el profesor, conjuntamente con sus estudiantes, deberá llevar a cabo el proceso de evaluación de las actitudes. una visión integral, situación que al docente debería preocuparle antes de cualquier otra consideración. Como dice Stenhouese: para evaluar hay que comprender. La evaluación no es cuestión de éxito o fracaso, el profesor debería ser un crítico, un estratega, y no un simple calificador (1984, citado en Suárez e Hinojoza, 2010).

Los exámenes extraordinarios son una oportunidad de aprendizaje en la que estudiantes y profesores convergen; la claridad, sistematización, pertinencia y eficiencia de los mismos es condición para el aprendizaje significativo, y esto está directamente relacionado con la intencionalidad, claridad, actitud y valores del profesor. No hay mejora sin evaluación; no hay aprendizaje sin reflexión. El tiempo destinado a la evaluación de los aprendizajes y al diseño de exámenes extraordinarios, trabajo o proyecto integral es una actividad medular que deben hacer los profesores en beneficio de los estudiantes.

\section{Fuentes de consulta}

Bertoni, A., Poggi, M. y Teobaldo, M. (2000). Evaluación. Nuevos significados para una práctica compleja. México: Norma, S. A.

Contreras, A. M. (2009). Elaboración de reactivos. Recuperado de: https://bit.ly/2Out4BG.

Coordinación de Calidad Académica (2014). Manual para la elaboración de exámenes escritos, entrega y exámenes orales. México: Universidad Anáhuac México Sur. Recuperado de: https://bit.ly/2RU1agv.

Flores de la Rosa, J. y García, M. A. (2018). Programa de nivelación académica 2018. México: UNAM. Recuperado de: https://bit.ly/2CKDGGo.

Suárez, E. L., Larrazabal, C. e Hinojoza, J. (2010). Diseño de un instrumento para evaluar la calidad de los exámenes escritos (teoría) de los estudiantes de medicina. Gac Med Bol, 33(2), 78-83. Recuperado de: https:// bit.ly/2Ckgvle.

UAA (2007). Modelo Educativo Institucional. Correo Universitario, séptima época, No. 15. [Primera reimpresión] 29 de mayo de 2015. México: Universidad Autónoma de Aguascalientes. Recuperado de: https://bit.ly/2OrlYxX.

UAA (2018). Reglamento General de Docencia de la Universidad Autónoma de Aguascalientes. Correo Universitario, octava época, No. 17, 1 de octubre de 2018. México: Universidad Autónoma de Aguascalientes. Recuperado de: https://bit.ly/2OrlYxX.

Wiggins, G. y McTighe, J. (2005). What it Backward Design? Understanding by design. (Cap. 1). Virginia, USA: ASCD. 